\title{
Determination of Infectious Bovine Viral Diarrhea Virus in Bovine Lung Lavages by a Combination of Virus Propagation in Cell Culture and Quantitative Real-Time PCR
}

\author{
Benjamin Zeitler and Ingrid Rapp \\ Labor Dr. Merk \& Kollegen GmbH, Beim Braunland 1, 88416 Ochsenhausen, Germany \\ Correspondence should be addressed to Benjamin Zeitler; zeitler@labormerk.de
}

Received 15 May 2013; Accepted 4 June 2013

Academic Editors: A. Doglio, A. Kfutwah, B. Kim, A. Mastino, and C. Risco

Copyright (C) 2013 B. Zeitler and I. Rapp. This is an open access article distributed under the Creative Commons Attribution License, which permits unrestricted use, distribution, and reproduction in any medium, provided the original work is properly cited.

\begin{abstract}
Material of bovine origin is often used in biotechnological applications. Bovine viral diarrhea virus (BVDV) is one of the major viral contaminants, and not only detection and inactivation but also quantification of the viral load in bovine starting material is required by the regulatory agencies. Here, we investigated combined virus propagation in cell culture and quantitative real-time PCR (qRT-PCR) for the applicability to detect and estimate low BVDV titers in bovine lung lavages, the source material for manufacturing pulmonary surfactant. qRT-PCR analyses of the crude lung lavages were performed and qRT-PCR calibration curves based on infective viral doses $\left(\mathrm{TCID}_{50} / \mathrm{mL}\right.$ ) were generated with a detection limit of $100 \mathrm{TCID}_{50} / \mathrm{mL}$. Lung lavages were inoculated on susceptible MDBK cells and cell culture samples were again analyzed by qRT-PCR. Immunofluorescence staining was performed to prove qRT-PCR results. Interestingly, initial BVDV contaminations in lung lavages were below qRT-PCR detection limit. An amplification step in cell culture enabled BVDV propagation to levels detectable by qRT-PCR. In comparison with the qRT-PCR calibration curve and control experiments with defined inoculation doses, the estimation of minor BVDV contaminations in lung lavages was possible. Both techniques can be successfully combined to estimate the viral load in dilute sample material.
\end{abstract}

\section{Introduction}

The genus Pestivirus of the Flaviviridae family includes the three important animal viruses classical swine fever virus (CSFV), border disease virus (BDV), and bovine viral diarrhea virus (BVDV) [1]. The latter is one of the most widespread cattle pathogens worldwide and two genotypes, each with a noncytopathic and cytopathic biotype, are known $[2,3]$. Infection of nonpregnant immunocompetent animals generally causes mild indisposition like ulceration of nose, mouth, or gastrointestinal mucosa resulting in continuous salivation, coughing, and diarrhea [4]. However, when pregnant animals become infected with BVDV, then abortion or malformation of the calf may occur. When infection takes place before the fourth month of gestation, persistently infected (PI) calves may be born. These animals are immunotolerant and develop normally in the absence of serious clinical symptoms of BVDV infection and may even be selected for breeding $[5,6]$. Severe mucosal disease, leading to death within weeks after breakout, can develop in PI animals after mutation of the noncytopathic BVDV into the cytopathic form. For healthy animals, PI animals represent an enormous risk of infection as they release large amounts of virus during their lifetime. In this way, BVDV guarantees preservation in the host population [5]. About 1\% of all cattle in BVDV contaminated regions are persistently infected. Furthermore, this virus is transmitted easily to other mammals, including sheep and goat $[4,7]$. Hence, a permanent risk of infection is present and efficient strategies to control BVDV infestation in prone regions have to be applied [8]. Particularly in countries and states where control-and-eradication campaigns do not rely on vaccination, also sensitive and simple test methods must be available to detect the virus in sample material of diverse origin [9].

Virus isolation and inoculation of cultured cells, followed by identification of the viral isolate by immunofluorescence 
or immunoperoxidase monolayer assay, are considered to be the gold standard for detection of BVDV [10]. If titers in the samples material are high enough, it is even possible to quantify the amount of virus by titration in susceptible cell lines [9]. Also, RT-PCR and antigen capture-ELISA are common techniques to identify virus infection directly. In particular, nucleic acid-based methods can be applied easily to quantify viral genomes in sample material. Nevertheless, the discrimination between uninfectious viral RNA and infectious viral particles is not possible as also free and defective genomes can serve as templates during the amplification reaction. Virus neutralization tests and anti-BVDV ELISA can be used to demonstrate BVDV infection indirectly by the detection of antibodies raised against the virus $[11,12]$. Of course, the test method has to be adapted to the available sample material, but independent of the technique these analyses cause costs and are often time consuming. For screening purposes and routinely inspection of entire herds or huge numbers of samples, sample pooling may be applied to reduce expenses. However, care must be taken, as with increasing pool size the danger of false negative results increases when assay sensitivity and specificity are too low [13]. On the other hand, pooling too few samples unnecessarily keeps the examination costs high $[10,14]$.

In addition to monitoring BVDV prevalence in cattle herds to control virus spreading, bovine raw material with intended use in industrial or pharmaceutical applications has to be analyzed for freedom of BVDV. According to the Guidelines of the International Conference on Harmonisation (ICH) or the European Medicines Agency (EMEA), pharmaceutical manufacturing processes must be able to remove or inactivate viral contaminations. Furthermore, independent of the production process the unprocessed bulk material has to be tested for viruses and the viral load has to be determined. Hence, not only the confirmation of presence or absence of viral nucleic acids, but also the identification and quantification of infectious particles are of special interest.

The present study was aimed at determining the viral load of BVDV in lung lavages of bovine origin. A combinational approach of virus propagation in cell culture and quantitative real-time PCR (qRT-PCR) was applied. Correctness of results was confirmed by an immunofluorescence test (IFT). Lungs of 80-100 slaughtered cattle were washed each with $80-100$ liters of a saline solution to obtain a lung lavage batch of about 8,000 liters that serves as source material for the production of pulmonary surfactant. By reduction of the surface tension, surfactants may prevent the collapse of alveoli of premature infants and allow the lung to inflate much more easily. The survival rate of premature infants suffering from infant respiratory distress syndrome can be increased dramatically by the application of pulmonary surfactants $[15,16]$. Commonly, bovine lung lavage batches are analyzed for virus contaminations by inoculation of susceptible cells, followed by staining with specific FITC-coupled antibodies. The pharmaceutical application of pulmonary surfactant implies intensive testing of raw materials for freedom of viruses. In particular, unidentified PI animals represent a source of virus contamination, and one single asymptomatic BVDV infected animal is sufficient to contaminate the entire batch. Cell culture-based assays take several days to weeks and are labor and cost intensive. Furthermore, to prevent degradation, crude lung lavages must be brought into a laborious manufacturing process immediately after extraction. At this point, results of the virus safety testing are not yet available, but if tested positive for pestiviruses, the whole pool has to be discarded. Albeit costs for lung lavage processing and by blocking the manufacturing line were unnecessarily generated.

Hence, we evaluated whether qRT-PCR might be used as cheap and fast alternative method to detect BVDV in bovine lung lavages. Regulations indicate the investigation of pharmaceutical raw material to determine the extent of viral burden. Thus, special emphasis was placed on the quantification of infectious BVDV particles, rather than solely on the detection of viral RNA in the sample material. For that purpose, we generated qRT-PCR calibration curves based on the $\mathrm{TCID}_{50} / \mathrm{mL}$ (tissue culture infective dose) by spiking BVDV strain NADL in previously BVDV negative tested lung lavages as well as in water to identify potential PCR inhibitors. The inclusion of defined calibrator samples in all analyses revealed validity and precise reproducibility of the calibration measurements. The limit of detection (LOD) was found to be $100 \mathrm{TCID}_{50} / \mathrm{mL}$. Interestingly, initial qRT-PCR results of three IFT BVDV positive lung lavages were negative. This indicated that viral titers in contaminated lung lavages were below qRT-PCR detection limit and terminated the original idea to replace the cell culture assay with qRT-PCR already at this point. However, another task was the viral load determination and we employed an amplification step in cell culture to propagate minimal BVDV contaminations to levels detectable by qRT-PCR. We monitored the increasing virus concentrations in lung lavage inoculated MDBK cells during two subcultivation steps. By comparison with the calibration curve, it was found that titers exceeded $10^{6.0} \mathrm{TCID}_{50} / \mathrm{mL}$ after two propagation steps, and results were confirmed by immunofluorescence staining. When cells were inoculated with a low BVDV strain NADL dose of $10 \mathrm{TCID}_{50} / \mathrm{mL}$ the measured Ct (Cycle threshold) values were only slightly above detection limit and, hence, the titer was far below titers in lung lavage inoculated MDBK cell cultures. Based on these experiments the titer of replication competent BVDV in analyzed lung washes can be estimated to range between 10 and $100 \mathrm{TCID}_{50} / \mathrm{mL}$. In all, these results highlight the sensitivity of cell culture-based BVDV detection and demonstrate the importance of accurate pool size determination to avoid false negatives in consequence of too dilute sample material. Furthermore, both techniques can be successfully combined to receive an impression of the viral load in dilute sample material representing a large sample pool. Thus, qRT-PCR can complement the qualitative IFT. Even approximate quantification of BVDV in unusual sample material like wash liquid of bovine lungs is conceivable when both methods are applied in combination.

\section{Material and Methods}

2.1. Cells and Virus. MDBK cells (CCL-22) were obtained from ATCC (Rockville, USA) and grown at $37^{\circ} \mathrm{C}$ and $5 \% \mathrm{CO}_{2}$ 
in Dulbecco's modified Eagle's medium (DMEM) supplemented with $7.5 \%(\mathrm{v} / \mathrm{v})$ fetal bovine serum (FBS), $100 \mathrm{units} / \mathrm{mL}$ penicillin, and $130 \mu \mathrm{g} / \mathrm{mL}$ streptomycin. Cells at $80 \%$ confluency were infected with BVDV type 1 (strain $\mathrm{NADL}$ ) and virus was propagated at $37^{\circ} \mathrm{C}$ and $5 \% \mathrm{CO}_{2}$ in maintenance medium (DMEM supplemented with $2.0 \%$ $(\mathrm{v} / \mathrm{v})$ FBS, 100 units $/ \mathrm{mL}$ penicillin, and $130 \mu \mathrm{g} / \mathrm{mL}$ streptomycin) for 3-5 d. Virus was released from infected cells by two freeze-thaw cycles and cell debris was removed from BVDV containing supernatant by centrifugation. MDBK cells in 96-well plates (Nunc, Germany) were inoculated with a dilution series from $10^{-1}$ to $10^{-8}$ with seven wells for each dilution and the titer of the $50 \%$ tissue culture infective dose of virus $\left(\mathrm{TCID}_{50}\right)$ was calculated according to the SpearmanKärber method [17, 18].

2.2. Bovine Lung Lavages. Lungs of slaughtered cattle were removed and each washed out with 80-100 liters of $0.9 \%$ $\mathrm{NaCl}$. After filtration to remove coarse impurities, $\mathrm{CaCl}_{2}$ $(7.9 \mathrm{~g} / \mathrm{L})$ was added and the liquid was cooled to $5^{\circ} \mathrm{C}$ until further processing. Washing of 80-100 lungs of healthy and veterinary controlled cattle resulted in approximately 8,000 liters of bovine lung lavage. Samples of $500 \mathrm{~mL}$ were drawn and stored below $-20^{\circ} \mathrm{C}$ until putative BVDV contaminations were analyzed by IFT and qRT-PCR. In this study, four lung lavage samples of the different production lots 1102 (BVDVnegative), 1104, 1108, and 1111 were investigated.

\subsection{BVDV Detection in Bovine Lung Lavages by IFT. Frozen} lung lavage samples were thawed to room temperature and filtered consecutively through $0.45 \mu \mathrm{m}$ and $0.22 \mu \mathrm{m}$ sterile syringe filters to remove crude particles and microbial contaminations. When MDBK cells in cell culture flasks reached $80 \%$ confluency, the medium was removed and cells were overlaid with $0.075 \mathrm{~mL} / \mathrm{cm}^{2}$ of the sterile filtrated lung lavages. Flasks were incubated at $37^{\circ} \mathrm{C}$ for $3 \mathrm{~h}$ before lung lavages were replaced with maintenance medium and incubation continued at $37^{\circ} \mathrm{C}$ and $5 \% \mathrm{CO}_{2}$. Medium was exchanged every 3-4 d and cells were subcultivated $7 \mathrm{dpi}$ and $14 \mathrm{dpi}$. Additionally, at each subcultivation step, cell cultivation on Lab-Tec chamber slides (Nunc, Germany) was initiated. After $7 \mathrm{~d}$, medium and upper structures were removed from the chamber slides; cells were washed in PBS and fixed in acetone for $10 \mathrm{~min}$ at room temperature. Air-dried microscope slides were incubated with BVDV type 1 and 2-reactive BVDVFITC-conjugate (VMRD, Inc., USA) for $30 \mathrm{~min}$ at $37^{\circ} \mathrm{C}$ in the dark, washed in carbonate buffer, and sealed with glycerol. Microscopic examination was performed under fluorescent light at 100-200x magnification with an Eclipse E400 fluorescence microscope (Nikon, Germany) and photographs were taken using the AxioCam MRC (Zeiss, Germany). Positive and negative controls were treated exactly the same way but were inoculated with either PBS or a dilute solution of BVDV strain NADL (100 $\left.\mathrm{TCID}_{50} / \mathrm{mL}\right)$.

2.4. RNA Isolation and $q R T-P C R$. Samples for PCR from cell culture or lung lavages were subjected to one freeze-thaw cycle to improve cell lysis, and RNA was extracted using the Ambion MagMAX-96 Blood RNA isolation kit (Applied Biosystems, Germany) and quantified with RiboGreen. An internal control emitting an additional signal during subsequent qRT-PCR was included during the RNA isolation procedure to identify potential PCR inhibitors and to assure equal sample processing. RNA concentration was adjusted to $15 \mathrm{ng} / \mu \mathrm{L}$ and $\mathrm{qRT}-\mathrm{PCR}$ analyses were performed using the cador BVDV RT-PCR Kit (Qiagen, Germany) and the Rotorgene 6000 RT-PCR cycler (Corbett, USA). $4 \mu \mathrm{L}$ samples (60 ng RNA) were used for PCR reactions and samples were measured in triplicate over 45 cycles. To avoid virus degradation, samples were always kept on ice and analyzed on the same day. Samples with no fluorescence signal (green channel) within 40 cycles were defined as BVDV-negative. Only measurements with the internal control (yellow channel) detected at $\mathrm{Ct}$ values around 30 were rated valid.

\subsection{Generation of $q R T-P C R$ Calibration Curves and BVDV} Quantification. Calibration curves were generated by spiking defined amounts of BVDV strain NADL in water and, to detect putative PCR inhibiting matrix effects, in lung lavage samples that were previously tested BVDV negative (lot 1102). The final BVDV titers in samples were $0,10^{1.0}, 10^{2.0}, 10^{2.5}, 10^{4.0}$, $10^{5.5}$, and $10^{7.0} \mathrm{TCID}_{50} / \mathrm{mL}$ and each sample was measured in triplicate. The calibration curves were constructed by plotting obtained Ct values over titers of infectious BVDV in the samples. BVDV contents in unknown samples were estimated by assigning measured $\mathrm{Ct}$ values with the respective virus titers.

\subsection{BVDV Detection in Bovine Lung Lavages and Cell Culture} Samples by RT-PCR. Unprocessed lung lavage samples were analyzed for the presence of BVDV as described in the RNA isolation and qRT-PCR section.

To estimate BVDV titers in lung lavage inoculated cell cultures, MDBK cells were treated as described for IFT and samples were taken at the first and second subcultivation steps $7 \mathrm{dpi}$ and $14 \mathrm{dpi}$, respectively. For that purpose, overlaying medium was removed cells were washed once with PBS and released by trypsin-EDTA treatment. Reaction was stopped by the addition of maintenance medium $(0.125 \mathrm{~mL} /$ $\mathrm{cm}^{2}$ ) before samples were drawn. RNA isolation, qRT-PCR, and BVDV quantification, in both medium supernatant and cell containing fraction, were performed as described in the RNA isolation and qRT-PCR section.

Control experiments were performed with defined inoculation doses of BVDV strain NADL. The medium of $80 \%$ confluent MDBK cells was removed and cells were overlaid with $0.0125 \mathrm{~mL} / \mathrm{cm}^{2}$ of the BVDV inoculum adjusted to 10 and $1000 \mathrm{TCID}_{50} / \mathrm{mL}$. After $1 \mathrm{~h}$ of incubation at $37^{\circ} \mathrm{C}$, maintenance medium was added and virus was allowed to propagate for $7 \mathrm{~d}$. Sampling and adjacent steps were performed as described for lung lavage treated cells.

2.7. Validation of Reproducibility of $q R T-P C R$ Measurements. Intra-assay variability was assessed by measuring each sample in triplicate. The coefficient of variation (CV) for each sample was calculated by dividing the standard deviation (SD) of results 1 to 3 by the triplicate mean and multiplying by 100 . 
The average of individual CVs displays the intra-assay CV [\%].

To prove the validity of the calibration curve and to ensure reproducible sample processing, low and high value calibrator samples $\left(10^{2.5}\right.$ and $\left.10^{5.5} \mathrm{TCID}_{50} / \mathrm{mL}\right)$ were included in the measurements when unknown samples were analyzed. In addition to the calibration curve generation, qRT-PCR analyses were performed at three different days and interassay variability was determined as follows. Individual means of low and high value samples, tested in total at four different days and in each case in triplicate, were used to calculate the overall means, SDs and CVs. The average of overall low and high value $\mathrm{CV}$ s reflects the interassay $\mathrm{CV}[\%]$.

\section{Results}

3.1. Generation of BVDV Calibration Curves. The possibility to replace cell culture-based BVDV detection in bovine lung lavages with qRT-PCR was evaluated during this study. Hence, in a first attempt, storage samples of the three previously BVDV-positive tested lung lavage lots 1104, 1108, and 1111 (data not shown) were analyzed for presence of BVDV by qRT-PCR using a commercial RT-PCR kit. Interestingly, although lung lavages were clearly identified BVDV-positive by immunofluorescence staining of inoculated MDBK cells, no BVDV specific signal was detected by qRT-PCR (see Supplementary Table S1, available online at http://dx.doi .org/10.5402/2013/751904). Nevertheless, as certainly infective BVDV particles must be present in the analyzed samples, the detection limit of the qRT-PCR was determined. Of special interest was the actual amount of viruses able to infect and propagate in cell culture. Thus, calibration samples of BVDV strain NADL were referred to the $\mathrm{TCID}_{50}$ rather than to free RNA. To detect putative PCR inhibitors present in the lung lavage, calibration curves were generated not only with BVDV spiked in negative lung lavage, but also with BVDV spiked in solely water. In addition, an internal control was included in the qRT-PCR kit by default to proof accurate assay performance. By plotting the obtained Ct values over corresponding BVDV strain NADL dilutions in lung lavage ranging from $10^{1.0}$ to $10^{7.0} \mathrm{TCID}_{50} / \mathrm{mL}$, a calibration curve with high linearity over $5 \log _{10}$ units could be constructed. In this experimental setup a detection limit of $100 \mathrm{TCID}_{50} / \mathrm{mL}$ was identified (Figure 1(a) and Supplementary Table S2). The correlation coefficient $R^{2}=0.9987$ indicated strong linearity of the calibration curve with the linear equation $y=-3.808 x+$ 47.281. Control experiments with BVDV spikes ranging from $10^{2.5}$ to $10^{7.0} \mathrm{TCID}_{50} / \mathrm{mL}$ in water, to exclude putative PCR-inhibiting matrix effects, resulted in a similar graph with the linear equation $y=-4.0427 x+48.963, R^{2}=0.9918$ (Figure 1(b) and Supplementary Table S3). The $\Delta$ slope was as little as 0.23 .

3.2. Intra-Assay Variability. Data of the calibration measurements were used to assess the intra-assay variability. Calculation of the coefficient of variation of both the pooled BVDV-spiked lung lavage and water samples as well as of the pooled internal controls revealed CVs of $0.66 \%$ and $0.41 \%$, respectively. This demonstrates high precision and accuracy of test results.

3.3. Replication of BVDV Strain NADL in MDBK Cells. When susceptible MDBK cells were inoculated with lung lavages 1104,1108 , and 1111, the contaminating BVDV could be propagated to levels allowing detection by IFT. But the failure to detect BVDV by qRT-PCR, although the LOD of $100 \mathrm{TCID}_{50} / \mathrm{mL}$ confirmed high sensitivity of the method, implied very low virus titers in the lavage liquids. To compare the degree of virus contamination, we monitored the virus replication in cell culture after infection with a low BVDV inoculum. MDBK cells were exposed to $10 \mathrm{TCID}_{50}$, similar to lung lavage inoculation, and viruses were allowed to multiply for $7 \mathrm{~d}$ before cells and medium were analyzed by qRT-PCR. Infection of cells with $1000 \mathrm{TCID}_{50}$ was performed in parallel. Inoculation with $10 \mathrm{TCID}_{50}$ was sufficient to permit BVDV to multiply in cell culture to detectable levels within one week. Based on previously generated qRT-PCR calibration curve, a virus titer of $10^{2.40} \mathrm{TCID}_{50} / \mathrm{mL}$ was measured in the cellular fraction. The amount of virus released into the medium supernatant was below LOD. Contrary, cells inoculated with $1000 \mathrm{TCID}_{50}$ were highly infected, displayed by a calculated titer of $10^{6.61} \mathrm{TCID}_{50} / \mathrm{mL}$, and the measured titer of $10^{5.30} \mathrm{TCID}_{50} / \mathrm{mL}$ in the culture supernatant implied that even high amount of virus was released from infected cells (Figure 2).

3.4. BVDV Propagation in Lung Lavage Inoculated Cell Cultures and Titer Estimation by $q R T-P C R$. The abovementioned results suggest that viral load in bovine lung lavages was below $100 \mathrm{TCID}_{50} / \mathrm{mL}$, as qRT-PCR did not produce any signal, but above $10 \mathrm{TCID}_{50} / \mathrm{mL}$, as this titer was sufficient to infect cell cultures and to allow BVDV to propagate to detectable levels within one week.

To gain further insights into the extent of BVDV contamination, we infected MDBK cells with the lung lavages. 7 and 14 dpi samples were drawn and again analyzed by qRT-PCR and, in addition, cultivation on chamber slides was initiated for IFT. Since BVDV titers were higher in cell containing samples than in medium supernatant (Figure 2), qRT-PCR was only performed with detached cells. Notably, already after one week lot 1104 inoculated cell culture was highly BVDV-positive, reflected by the calculated titer of $10^{6.56} \mathrm{TCID}_{50} / \mathrm{mL}$ (Figure 3). Probably, BVDV content in the lung lavage was only slightly below qRT-PCR detection limit of $100 \mathrm{TCID}_{50} / \mathrm{mL}$ and, thus, favored BVDV propagation. This finding was in accordance with the primary qRT-PCR analysis of the lung lavage lot 1104. Although in sum the initial measurement was rated BVDV-negative, in one of the three replicates a Ct value of 40.23 indicated the presence of BVDV (Supplementary Table S1). After another week of virus multiplication, the BVDV titer in lot 1104 inoculated cell culture was $10^{6.29} \mathrm{TCID}_{50} / \mathrm{mL}$ and, hence, remained more or less constant (Supplementary Table S5). Possibly, virus propagation reached a plateau reflecting maximal BVDV multiplication rate in cell culture. In lot 1108 and lot 1111 inoculated cell cultures the initial BVDV titers were apparently lower and 


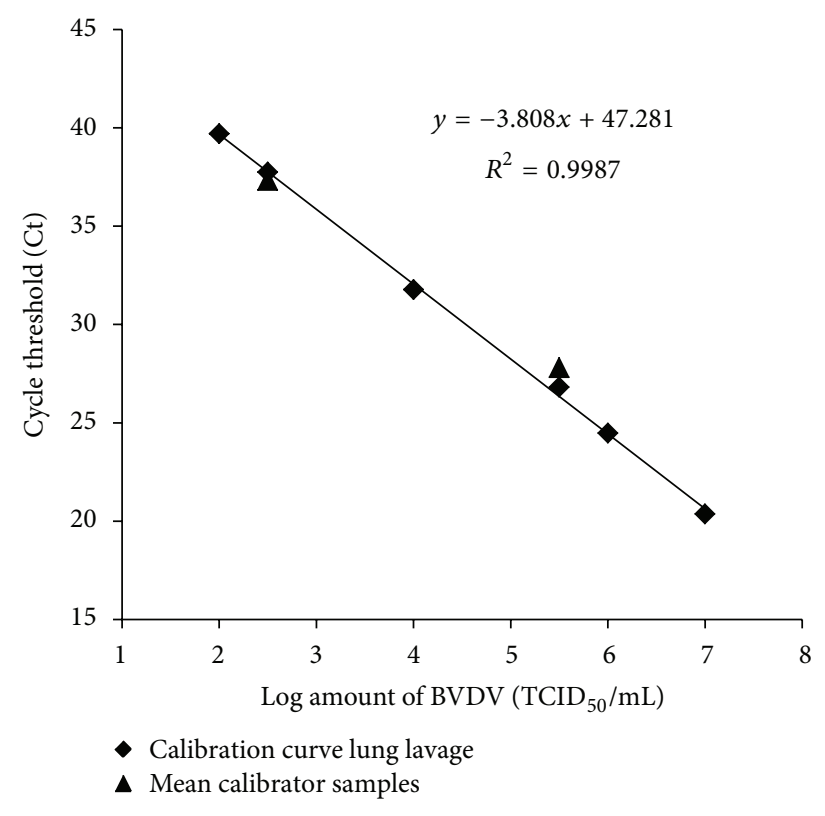

(a)

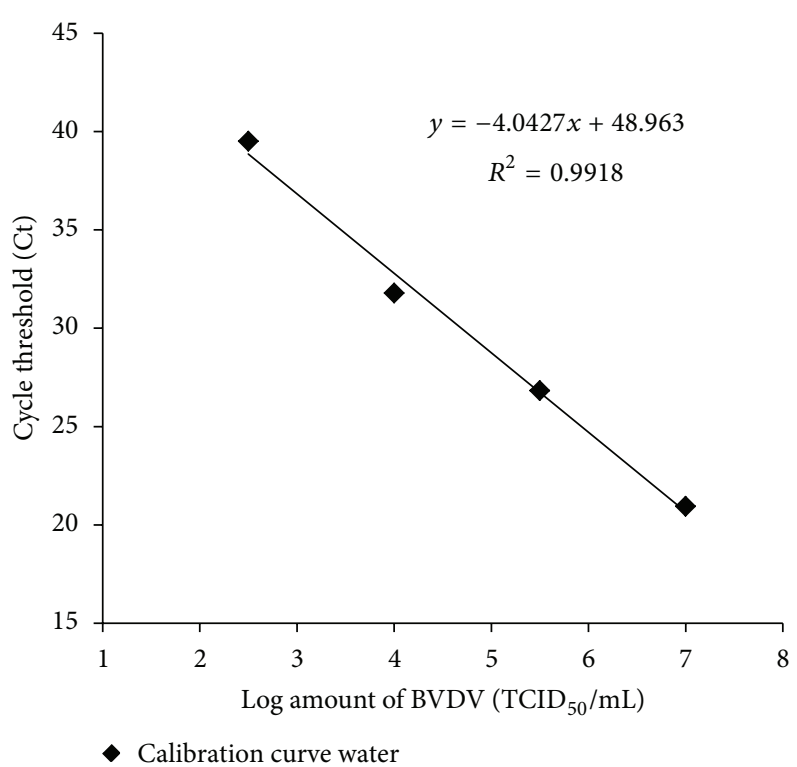

(b)

FIGURE 1: BVDV calibration curves. (a) Calibration curve generated by RT-PCR with the cador BVDV RT-PCR Kit using virus spiked lung lavage in the range from $10^{2.0}$ to $10^{7.0} \mathrm{TCID}_{50} / \mathrm{mL}$. Black triangles indicate means of the Ct values of calibrator samples included in further measurements of unknown samples to proof validity of the calibration curve. (b) Calibration curve according to (a) but BVDV spiked in water in the range from $10^{2.5}$ to $10^{7.0} \mathrm{TCID}_{50} / \mathrm{mL}$. Shown are the mean Ct values of triplicate measurements. Raw data, including standard deviations (SD) and Ct values of the internal control, are presented in Supplementary Tables S2 and S3.

ranged $7 \mathrm{dpi}$ at $10^{4.94}$ and $10^{5.68} \mathrm{TCID}_{50} / \mathrm{mL}$, respectively. A clear kinetics of virus replication can be derived and $14 \mathrm{dpi}$ the titers increased to $10^{6.36}$ and $10^{6.67} \mathrm{TCID}_{50} / \mathrm{mL}$, respectively, (Figure 3 and Supplementary Table S5). In comparison to the detected BVDV titer of $10^{2.40} \mathrm{TCID}_{50} / \mathrm{mL}$ in cell culture inoculated with $10 \mathrm{TCID}_{50} / \mathrm{mL}$ (Figure 2), the measured titers in cell cultures inoculated with lung lavage lots 1108 and 1111 were substantially higher. Consequently, it can be assumed that also in these lung lavages the titers of infectious BVDV particles were closer to $100 \mathrm{TCID}_{50} / \mathrm{mL}$ than to $10 \mathrm{TCID}_{50} / \mathrm{mL}$.

3.5. Interassay Variability. During all measurements low and high value calibrator samples, adjusted to $10^{2.5}$ and $10^{5.5} \mathrm{TCID}_{50} / \mathrm{mL}$, were included to control applicability and validity of previously determined calibration curve. The mean $\mathrm{Ct}$ of three calibrator measurements of samples with $10^{2.5} \mathrm{TCID}_{50} / \mathrm{mL}$ was found to be $37.33 \pm 1.13$ and differed only 0.41 cycles from the calibration curve. Calibrator samples containing $10^{5.5} \mathrm{TCID}_{50} / \mathrm{mL}$ had a mean Ct of $27.80 \pm 0.07$ and the difference to the calibration value was as little as 0.98 cycles (Figure 1(a) and Supplementary Table S6). Based on these calibrator samples the interassay variability could be evaluated and the calculated CV of $1.69 \%$ indicated strong assay reproducibility. Thus, validity of the calibration curve was definitely shown. by IFT. To proof that qRT-PCR results can be really related to infective, replicating viruses, the lung lavage infected MDBK cells were sown on chamber slides for subsequent immunostaining with FITC-coupled anti-BVDV-antibodies (Figure 4 and Supplementary Figure S1). All lung lavage infected cell cultures were clearly BVDV-positive in the IFT. However, an estimate of the viral load based on fluorescence intensity was certainly not possible either after one or after two weeks of cultivation.

\section{Discussion}

The efficient monitoring and detection of BVDV infection in cattle is an important issue to control the spreading of this ubiquitous pathogen and to support eradication campaigns [8]. In addition, bovine material with intended use in diagnostic or pharmaceutical applications must be strictly analyzed for virus contaminations. Moreover, viral safety guidelines claim the testing of unprocessed bulk material. In all cases, these examinations are related to costs and, of special importance for pharmaceutical production, they delay manufacturing processes. To reduce costs for virus safety testing, the analyses of sample pools may be applied but require sensitive and reproducible assays. For this reason, we evaluated the potential of quantitative real-time PCR to replace labor- and time-intensive cell culture-based BVDV detection with IFT in bovine lung lavages. There are several reports about similar studies comparing different methods for BVDV detection, but mostly bovine blood, serum, or tissue samples were investigated. Laamanen et al. [19], for example, compared reverse 


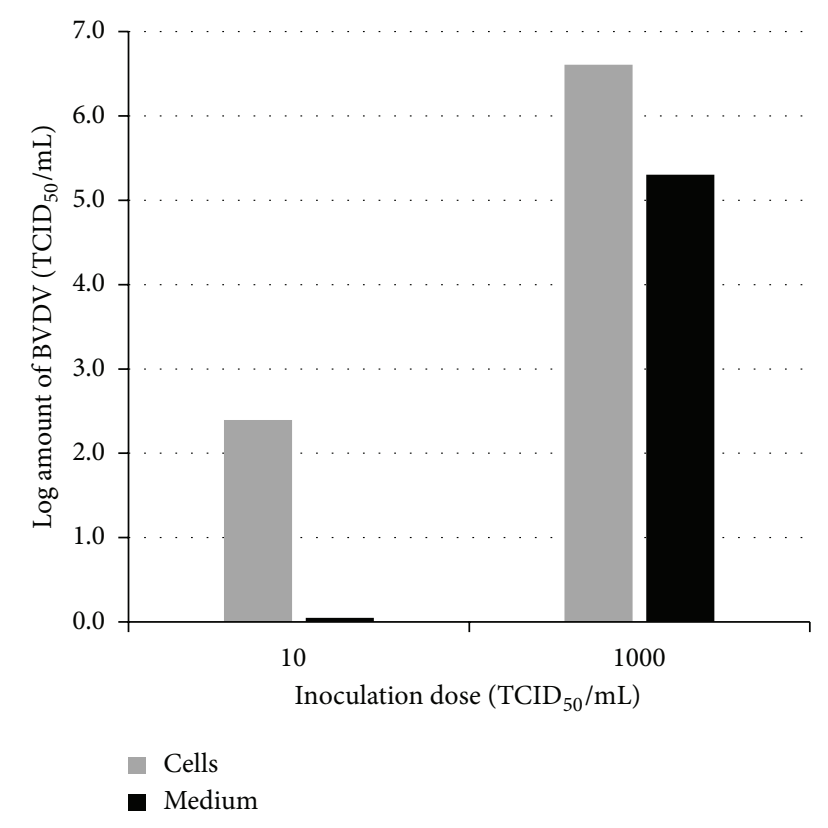

FIGURE 2: Replication of BVDV strain NADL in MDBK cells. 7 dpi medium and cells were analyzed by RT-PCR and BVDV was quantified according to the calibration curve shown in Figure 1(a). Raw data, including standard deviations (SD) and Ct values of the internal control, are presented in Supplementary Table S4.

transcription PCR and virus isolation-immunoperoxidase test (IPX) for detection of BVDV in serum samples. Both methods were well suited for identification of BVDV infection when dilutions of $10^{-3}-10^{-4}$ in the IPX and dilutions of $10^{-2}-10^{-3}$ in the reverse transcription PCR were used. In accordance with the present study, the cell-based assay was slightly more sensitive than the PCR. However, an important point is discussed justifying the detection of viral RNA for BVDV analyses. By reverse transcription-PCR nucleic acids are also detected in samples in which virus may be inactivated either by the presence of neutralizing antibodies or improper sample handling. Of special interest during the present study was the estimation of infective viral BVDV load in lung lavages of bovine origin and, thus, we first generated a calibration curve based on replicating viral particles spiked into the BVDV-negative lung lavage 1102. The generated curve was linear over the entire measured range from $10^{2.0}$ to $10^{7.0} \mathrm{TCID}_{50} / \mathrm{mL}$. It was not possible to detect viruses efficiently in samples containing less than $100 \mathrm{TCID}_{50} / \mathrm{mL}$ and, therefore, the detection limit was set at this level. A onestep SYBR Green I quantitative real-time PCR assay for the detection of BVDV type 1 in cell culture samples was also developed by Zhang et al. [20]. In a comparable experimental setup, they constructed an RNA standard curve using in vitro transcripts of a BVDV cDNA template ranging from $10^{2.0}$ to $10^{7.0}$ copies $/ \mathrm{mL}$. The relation of Ct values of serial dilutions of titrated virus to the RNA standard curve revealed the detection limit at the $10^{-5}$ virus dilution, equal to $8.79 \times 10^{2}$ copies/ $\mathrm{mL}$ of BVDV RNA. Compared to conventional RT-PCR, the one-step SYBR Green I RT-PCR assay was 10-fold more sensi-

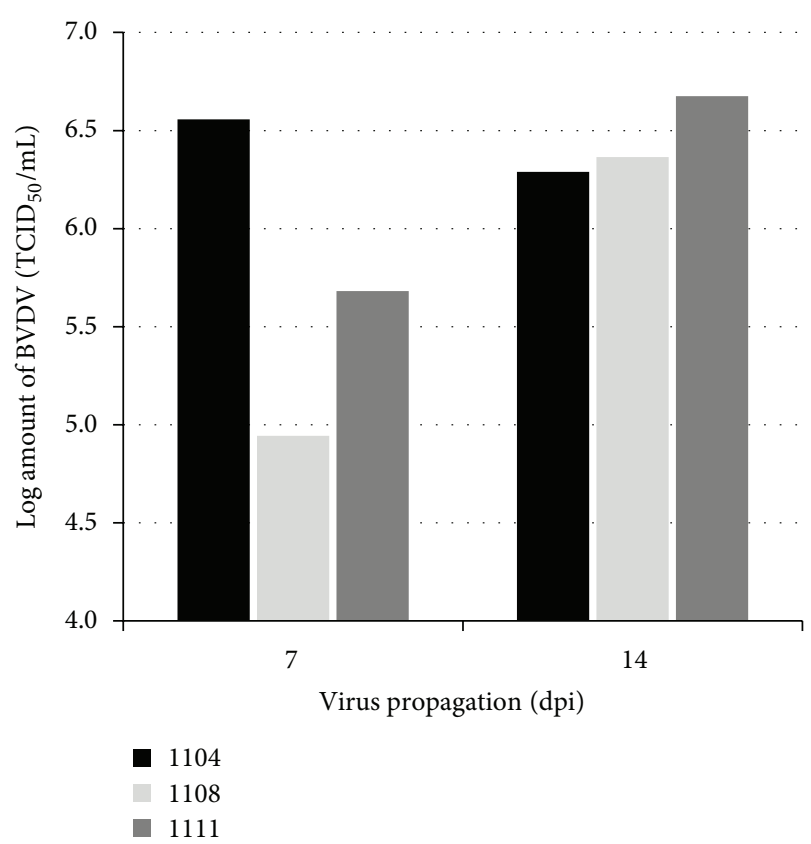

FIGURE 3: BVDV titer estimation in lung lavage inoculated MDBK cells. 7 and 14 dpi cells were released by trypsin, analyzed by RT-PCR and BVDV was quantified according to the calibration curve shown in Figure 1(a). Raw data, including standard deviations (SD) and Ct values of the internal control, are presented in Supplementary Table S5.

tive. In addition to RNA, recombinant plasmid DNA containing viral sequences can be used for calibration curve generation, as it was done by Kosinova et al. [21]. They found only slight differences between cDNA- and RNA-based calibration curves and analyzed the viral BVDV load in bulk milk samples, serum samples, and in a batch of calf serum for use in cell culture. Interestingly, even in the latter they found 23.27 BVDV copies/ $\mu \mathrm{L}$ when $\mathrm{Ct}$ values were extrapolated to the cDNA standard curve. Most batches of FCS contain noninfectious viral RNA detectable by RT-PCR. Hence, the use of cell cultures to amplify low amounts of infectious virus also increases the risk of contamination. The negative controls in the present study were uninfected MDBK cells and FCS containing medium supernatant. In both samples, no BVDV-specific signal was detected by qRT-PCR. In addition, the BVDV strain NADL used as spikes for generation of the calibration curves was propagated in FCS containing medium. In high dilutions, no BVDV-specific signal was detected by qRT-PCR. Based on these findings, we can assume that neither uninfected MDBK cells interfere with the assay nor BVDV derived nucleic acids were present in the culture medium components. The use of real-time PCR assays for pathogen detection provides several advantages compared to cell culture-based assays, like reduced costs, the wide dynamic range of sample analysis, the quantitative rather than only qualitative capacity, and little interassay variation [22]. The latter was also demonstrated in our study and the interassay CV of $1.69 \%$ indicated strong reproducibility of the assay and validity of the generated calibration curves and measurements. With regard to cost reduction, Yan et al. [23] 
7 dpi
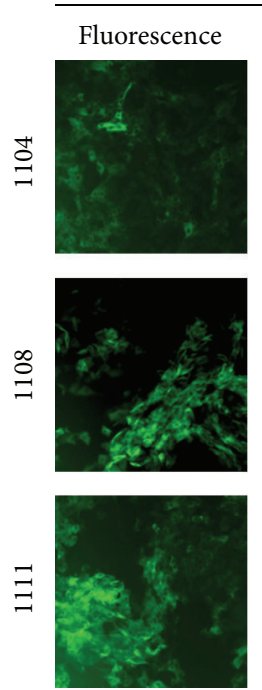

Bright field
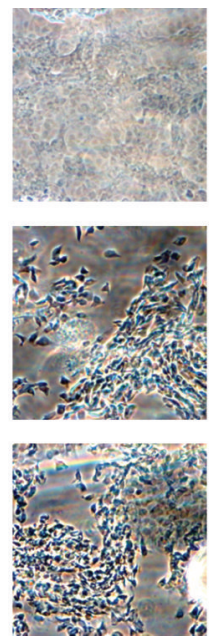
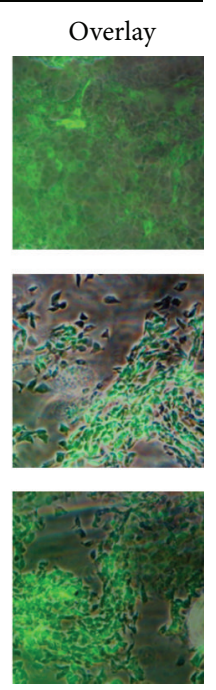

14 dpi

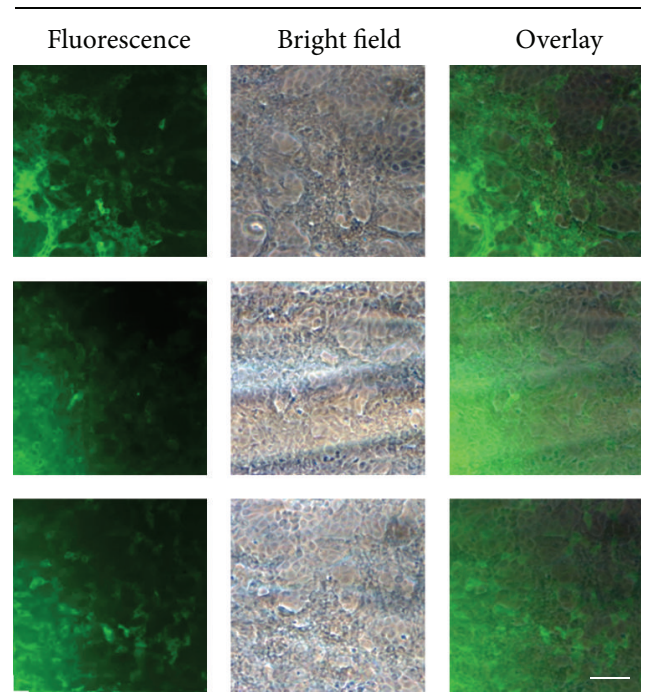

FIGURE 4: BVDV detection in lung lavage inoculated MDBK cells by IFT with anti-BVDV FITC-coupled antibodies. Cell cultures were inoculated with lung lavages 1104, 1108, and 1111 and growth on chamber slides was initiated 7 and 14 dpi, respectively. Microscopic examination was performed with an Eclipse E400 fluorescence microscope (Nikon, Germany) at 100-200x magnification under fluorescent light. Photographs were taken using the AxioCam MRC and analyzed with AxioVision Rel 4.7 (Zeiss, Jena, Germany). Scale bar $100 \mu \mathrm{m}$.

evaluated the combination of RT-PCR and antigen captureELISA (ACE) for the detection of BVDV PI animals in exposed herds. Theoretically, cost reduction by the combinational approach ranged from $61 \%$ to $94 \%$, compared with testing individual samples by ACE, RT-PCR, or immunohistochemistry. For determination of RT-PCR sensitivity, they used RNA extracted from serial dilutions of BVDV-1 and BVDV-2 stocks or serial dilutions of in vitro transcribed RNA. The defined detection limits based on virus concentrations were $10^{1.33} \mathrm{TCID}_{50}$ per $25 \mu \mathrm{L}$ reaction for BVDV-1 and $10^{2.0} \mathrm{TCID}_{50}$ per $25 \mu \mathrm{L}$ reaction for BVDV-2. Interestingly, detection limits determined with in vitro transcribed RNA spiked in negative ear notch samples as matrix material were even higher. For BVDV-1 $10^{2.0}$ copies per $25 \mu \mathrm{L}$ reaction and for BVDV-2 $10^{3.0}$ copies per $25 \mu \mathrm{L}$ reaction are reported. The results of RT-PCR and ACE were moderately correlated. In our study, a detection limit of $10^{2.0} \mathrm{TCID}_{50} / \mathrm{mL}$ was identified. Accordingly, the presented RT-PCR assay is actually more sensitive.

However, it must be kept in mind that several technical and biological challenges might arise when qRT-PCR is applied to quantify viral RNA in unknown sample material. Sample collection from different origins or processing at different days and under varying conditions may result in a certain degree of sample-to-sample variation. In particular, RNA extraction, efficiency of reverse transcription, or the presence of putative PCR-inhibitors can significantly affect sensitivity of this technique. Young et al. [24] also addressed these points, and they used a second RNA virus, Canine Enteric Coronavirus (CECov), as external RNA reference for normalization. Samples were spiked with a known amount of CECov prior to processing to allow correction of differences in the efficiency of RNA extraction and RT-PCR. Their assay was employed to detect acute BVDV infection in whole blood samples from cattle, and the detection limit of $2.1 \times$ $10^{1.0} \mathrm{TCID}_{50}$ indicated high sensitivity of the method. Degradation of RNA by almost everywhere present RNAses is a major risk when dealing with the detection of viral RNA genomes. Hence, careful sample handling is of great concern and BVDV RNA in serum samples was shown to be degraded within $48 \mathrm{~h}$ when stored at ambient temperature. Decreasing storage temperature to $4^{\circ} \mathrm{C}$ prolonged RNA stability to at least $72 \mathrm{~h}$, whereas rate of degradation was strongly dependent on initial virus titer and purity of serum [25]. When tissue samples were held at $4^{\circ} \mathrm{C}$ for up to one week prior to formalin fixation and subsequent BVDV detection by real-time PCR, the cycle thresholds remained relatively unchanged. However, for samples stored at room temperature prior to fixation and qRT-PCR the cycle numbers increased and at $96 \mathrm{~h} \mathrm{BVDV}$ was not detected anymore [26]. The lung lavages investigated in the present study were immediately chilled to $4^{\circ} \mathrm{C}$ after initial production and samples for analyses were frozen at $-20^{\circ} \mathrm{C}$ or below within $24 \mathrm{~h}$. In addition, sample preparation for qRT-PCR measurements was strictly performed at low temperatures and, therefore, virus degradation or loss of detectable viral RNA due to faulty sample handling can be considered negligible. The presence of potential PCRinhibitors in lung lavages and as consequence the generation of false negative RT-PCR results can also be excluded, as BVDV spiked samples for calibration measurements were prepared in BVDV negative lung lavage as well as in water. Both derived calibration curves were highly linear over the entire measured range. Contrary to the above mentioned investigations, the present study was aimed at quantifying infectious BVDV in sample material containing viral load de facto below qRT-PCR detection limit. Moreover, as lung 
lavage lots were manufactured by processing lungs of $80-100$ cattle, such material can be considered exemplarily as large sample pool. A virus propagation step in cell culture was employed to allow virus to multiply to detectable levels before infected cells were analyzed by RT-PCR. We first identified $10 \mathrm{TCID}_{50}$ to be sufficient for initiation of viral infection of MDBK cells, and 7 dpi BVDV determination in the cellular fraction by qRT-PCR revealed a titer of $10^{2.40} \mathrm{TCID}_{50} / \mathrm{mL}$. These findings allowed BVDV titer estimation in lung lavage inoculated cell culture samples. It was most apparently after one week of virus propagation that lung lavages differed in BVDV titers. In cells inoculated with lot 1104, the titer of $10^{6.56} \mathrm{TCID}_{50} / \mathrm{mL}$ was detected, whereas in lot 1108 and lot 1111 inoculated cells lower titers of $10^{4.94} \mathrm{TCID}_{50} / \mathrm{mL}$ and $10^{5.68} \mathrm{TCID}_{50} / \mathrm{mL}$, respectively, were extrapolated. Therefore, virus contamination in lot 1104 was probably slightly below qRT-PCR detection limit of $100 \mathrm{TCID}_{50} / \mathrm{mL}$, but BVDV titers in lot 1108 and lot 1111 likely ranged between 10 and $100 \mathrm{TCID}_{50} / \mathrm{mL}$. After another subcultivation period of $7 \mathrm{~d}$, BVDV titers were rather equal in all analyzed samples and probably reached the plateau of maximal virus propagation upon given cell culture conditions. An estimate of BVDV load in lung lavages by classical cell culture-based IFT was definitely not possible, although this approach in general was shown to be more sensitive than qRT-PCR. However, limitations of the present approach must be mentioned. Strain NADL is a cytopathic BVDV strain, and it is known that cytopathic viruses produce substantially more viral RNA inside a cell than their noncytopathic counterparts $[27,28]$. Furthermore, large variability in the replication of viral RNA between various BVDV strains was reported $[29,30]$. Therefore, the strict extrapolation of the cytopathic NADL strain to the unknown strains present in the lung lavage samples is subject to some restrictions. The interpretation might be verified in future studies by applying various noncytopathic BVDV strains in the assay. Nevertheless, it should be obvious that conclusions on the initial amount of infectious BVDV in unknown samples can be drawn by monitoring the virus replication kinetics in cell culture as it was done in this study. Finally, comparison to the growth characteristics of a known reference strain will enable titer estimation.

Despite the successful implementation of regional eradication campaigns, BVDV infections of cattle remain a source of severe economic loss not only for producers, but also for downstream industries [8]. Besides prevention of infection by vaccination, currently no specific therapeutic treatments are available. Nevertheless, immunocompetent animals may recover depending on the severity of the disease and the administered supportive therapy. The latter may rely on antiviral treatments, and currently several antiviral compounds are investigated [31,32]. For example, the aromatic compound DB772 inhibits diverse pestiviruses including BVDV already at concentrations $>0.20 \mu \mathrm{M}$. As no cytotoxicity was detected at these concentrations, the compound may represent a potential preventative or therapeutic agent for diverse pestiviral infections [33]. Only recently a high throughput antiBVDV drug screening assay, based on a recombinant GFP reporter virus, was presented to be capable of identifying inhibitors of different steps in the BVDV lifecycle [34]. How- ever, the identification of existent infections of cattle and the rigorous specifications for testing of bovine raw material clearly demonstrate the need for sensitive and economical detection systems. Pathogen detection by qRT-PCR has become common for quantification of RNA targets and determination of viral load in clinical samples [22]. In the present study, we combined cell culture-based IFT and qRT-PCR to estimate BVDV contamination in bovine lung lavages that was initially below RT-PCR detection limit. This approach may be used to analyze large sample pools and combines high sensitivity of the cell culture-based technique as well as the quantitative character of qRT-PCR.

\section{Conflict of Interests}

The authors declare that they have no conflict of interests.

\section{Acknowledgments}

The authors thank Dr. Apollonia Nagler for providing the lung lavages and for critical reading of the paper. They also feel grateful to Dr. Vital Wohlgensinger for help during qRTPCR measurements. Special thanks are due to Bernadette Eberle, Margret Jehle, and Julia Tilkorn for the perfect technical assistance during cell culture experiments. This study was funded by a private sponsor. Both authors are employed at the Labor Dr. Merk \& Kollegen GmbH (Ochsenhausen, Germany).

\section{References}

[1] S. Modrow, D. Falke, and U. Truyen, Molekulare Virologie, Spektrum Akademischer, Heidelberg, Germany, 2nd edition, 2003.

[2] J. C. Baker, "Bovine viral diarrhea virus: a review," Journal of the American Veterinary Medical Association, vol. 190, no. 11, pp. 1449-1458, 1987.

[3] J. F. Ridpath, "Bovine viral diarrhea virus," in Encyclopedia of Virology, D. H. Bamford, C. Calisher, A. J. Davison et al., Eds., pp. 374-380, Academic Press, Oxford, UK, 3rd edition, 2008.

[4] P. Leyssen, E. De Clercq, and J. Neyts, "Perspectives for the treatment of infections with Flaviviridae," Clinical Microbiology Reviews, vol. 13, no. 1, pp. 67-82, 2000.

[5] E. Peterhans, C. Bachofen, H. Stalder, and M. Schweizer, "Cytopathic bovine viral diarrhea viruses (BVDV): emerging pestiviruses doomed to extinction," Veterinary Research, vol. 41, no. 6, article 44, 2010.

[6] S. D. Goens, "The evolution of bovine viral diarrhea: a review," Canadian Veterinary Journal, vol. 43, no. 12, pp. 946-954, 2002.

[7] F. S. Dehkordi, "Prevalence study of Bovine viral diarrhea virus by evaluation of antigen capture ELISA and RT-PCR assay in Bovine, Ovine, Caprine, Buffalo and Camel aborted fetuses in Iran," AMB Express, vol. 1, no. 1, article 32, 2011.

[8] J. F. Ridpath, "Bovine viral diarrhea virus: global status," Veterinary Clinics of North America, vol. 26, no. 1, pp. 105-121, 2010.

[9] H. Houe, A. Lindberg, and V. Moennig, "Test strategies in bovine viral diarrhea virus control and eradication campaigns in Europe," Journal of Veterinary Diagnostic Investigation, vol. 18 , no. 5, pp. 427-436, 2006. 
[10] M. A. Edmondson, M. D. Givens, P. H. Walz, J. A. Gard, D. A. Stringfellow, and R. L. Carson, "Comparison of tests for detection of bovine viral diarrhea virus in diagnostic samples," Journal of Veterinary Diagnostic Investigation, vol. 19, no. 4, pp. 376-381, 2007.

[11] H. Houe, "Epidemiological features and economical importance of bovine virus diarrhoea virus (BVDV) infections," Veterinary Microbiology, vol. 64, no. 2-3, pp. 89-107, 1999.

[12] A. Özkul, K. Yeşilbağ, and I. Burgu, "Comparison of four diagnostic techniques for detecting Bovine Virus Diarrhoea Virus (BVDV) in buffy coat samples after long-term storage," Turkish Journal of Veterinary and Animal Sciences, vol. 26, no. 5, pp. 1043-1048, 2002.

[13] J. Laureyns, S. Ribbens, and A. de Kruif, "Control of bovine virus diarrhoea at the herd level: reducing the risk of false negatives in the detection of persistently infected cattle," Veterinary Journal, vol. 184, no. 1, pp. 21-26, 2010.

[14] K. Singh, M. M. Miller, L. J. Kohrt, G. Scherba, E. F. Garrett, and R. L. Fredrickson, "Development of a novel diagnostic test for detection of bovine viral diarrhea persistently infected animals using hair," Journal of Veterinary Science, vol. 12, no. 3, pp. 295297, 2011.

[15] J. R. Glasser and R. K. Mallampalli, "Surfactant and its role in the pathobiology of pulmonary infection," Microbes and Infection, vol. 14, no. 1, pp. 17-25, 2012.

[16] D. F. Willson and R. H. Notter, "The future of exogenous surfactant therapy," Respiratory Care, vol. 56, no. 9, pp. 1369-1386, 2011.

[17] C. Spearman, "The method of "right and wrong cases" ("constant stimuli") without Gauss's formulae," British Journal of Psychology, vol. 2, no. 3, pp. 227-242, 1908.

[18] G. Kärber, "Beitrag zur kollektiven Behandlung pharmakologischer Reihenversuche," Naunyn-Schmiedebergs Archiv für Experimentelle Pathologie und Pharmakologie, vol.162, no. 4, pp. 480-483, 1931.

[19] U. I. Laamanen, E. P. Neuvonen, E. M. Yliviuhkola, and P. M.-L. Veijalainen, "Comparison of RT-PCR assay and virus isolation in cell cultures for the detection of bovine viral diarrhoea virus (BVDV) in field samples," Research in Veterinary Science, vol. 63, no. 3, pp. 199-203, 1997.

[20] N. Zhang, Z. Liu, Q. Han et al., "Development of one-step SYBR Green real-time RT-PCR for quantifying bovine viral diarrhea virus type-1 and its comparison with conventional RT-PCR," Virology Journal, vol. 8, article 374, 2011.

[21] E. Kosinova, I. Psikal, B. Robesova, and K. Kovarcik, "Real-time PCR for quantitation of bovine viral diarrhea virus RNA using SYBR Green I fluorimetry," Veterinary Medicine, vol. 52, no. 6, pp. 253-261, 2007.

[22] S. A. Bustin and R. Mueller, "Real-time reverse transcription PCR (qRT-PCR) and its potential use in clinical diagnosis," Clinical Science, vol. 109, no. 4, pp. 365-379, 2005.

[23] L. Yan, S. Zhang, L. Pace, F. Wilson, H. Wan, and M. Zhang, "Combination of reverse transcription real-time polymerase chain reaction and antigen capture enzyme-linked immunosorbent assay for the detection of animals persistently infected with Bovine viral diarrhea virus," Journal of Veterinary Diagnostic Investigation, vol. 23, no. 1, pp. 16-25, 2011.

[24] N. J. Young, C. J. Thomas, M. E. Collins, and J. Brownlie, "Realtime RT-PCR detection of Bovine Viral Diarrhoea virus in whole blood using an external RNA reference," Journal of Virological Methods, vol. 138, no. 1-2, pp. 218-222, 2006.
[25] D. Weinstock, B. Bhudevi, and A. E. Castro, "Single-tube singleenzyme reverse transcriptase PCR assay for detection of bovine viral diarrhea virus in pooled bovine serum," Journal of Clinical Microbiology, vol. 39, no. 1, pp. 343-346, 2001.

[26] B. Bhudevi and D. Weinstock, "Detection of bovine viral diarrhea virus in formalin fixed paraffin embedded tissue sections by real time RT-PCR (Taqman)," Journal of Virological Methods, vol. 109, no. 1, pp. 25-30, 2003.

[27] Y. Li and J. McNally, "Characterization of RNA synthesis and translation of bovine viral diarrhea virus (BVDV)," Virus Genes, vol. 23, no. 2, pp. 149-155, 2001.

[28] V. B. Vassilev and R. O. Donis, "Bovine viral diarrhea virus induced apoptosis correlates with increased intracellular viral RNA accumulation," Virus Research, vol. 69, no. 2, pp. 95-107, 2000.

[29] D. Yamane, K. Kato, Y. Tohya, and H. Akashi, "The doublestranded RNA-induced apoptosis pathway is involved in the cytopathogenicity of cytopathogenic Bovine viral diarrhea virus," Journal of General Virology, vol. 87, no. 10, pp. 2961-2970, 2006.

[30] S. A. La Rocca and T. Sandvik, "A short target real-time RT-PCR assay for detection of pestiviruses infecting cattle," Journal of Virological Methods, vol. 161, no. 1, pp. 122-127, 2009.

[31] B. W. Newcomer, M. S. Marley, P. K. Galik et al., "Antiviral treatment of calves persistently infected with bovine viral diarrhoea virus," Antiviral Chemistry \& Chemotherapy, vol. 22, no. 4, pp. 171-179, 2012.

[32] M. T. A. Salim, Y. Goto, T. Hamasaki et al., "Highly potent and selective inhibition of bovine viral diarrhea virus replication by $\gamma$-carboline derivatives," Antiviral Research, vol. 88, no. 3, pp. 263-268, 2010.

[33] B. W. Newcomer, M. S. Marley, J. F. Ridpath et al., "Efficacy of an antiviral compound to inhibit replication of multiple pestivirus species," Antiviral Research, vol. 96, no. 2, pp. 127-129, 2012.

[34] Z.-C. Fan and R. C. Bird, "Development of a reporter bovine viral diarrhea virus and initial evaluation of its application for high throughput antiviral drug screening," Journal of Virological Methods, vol. 180, no. 1-2, pp. 54-61, 2012. 

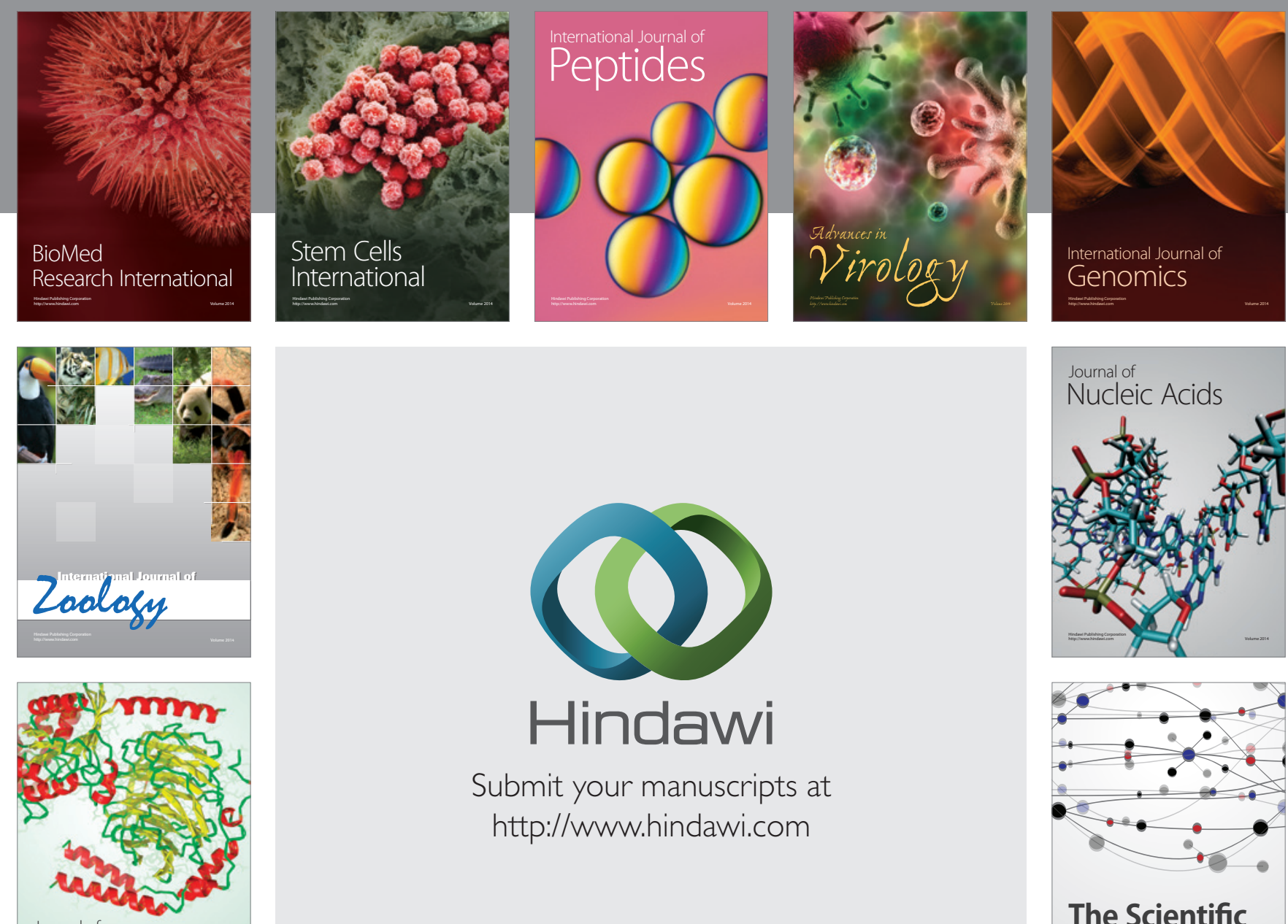

Submit your manuscripts at

http://www.hindawi.com

Journal of
Signal Transduction
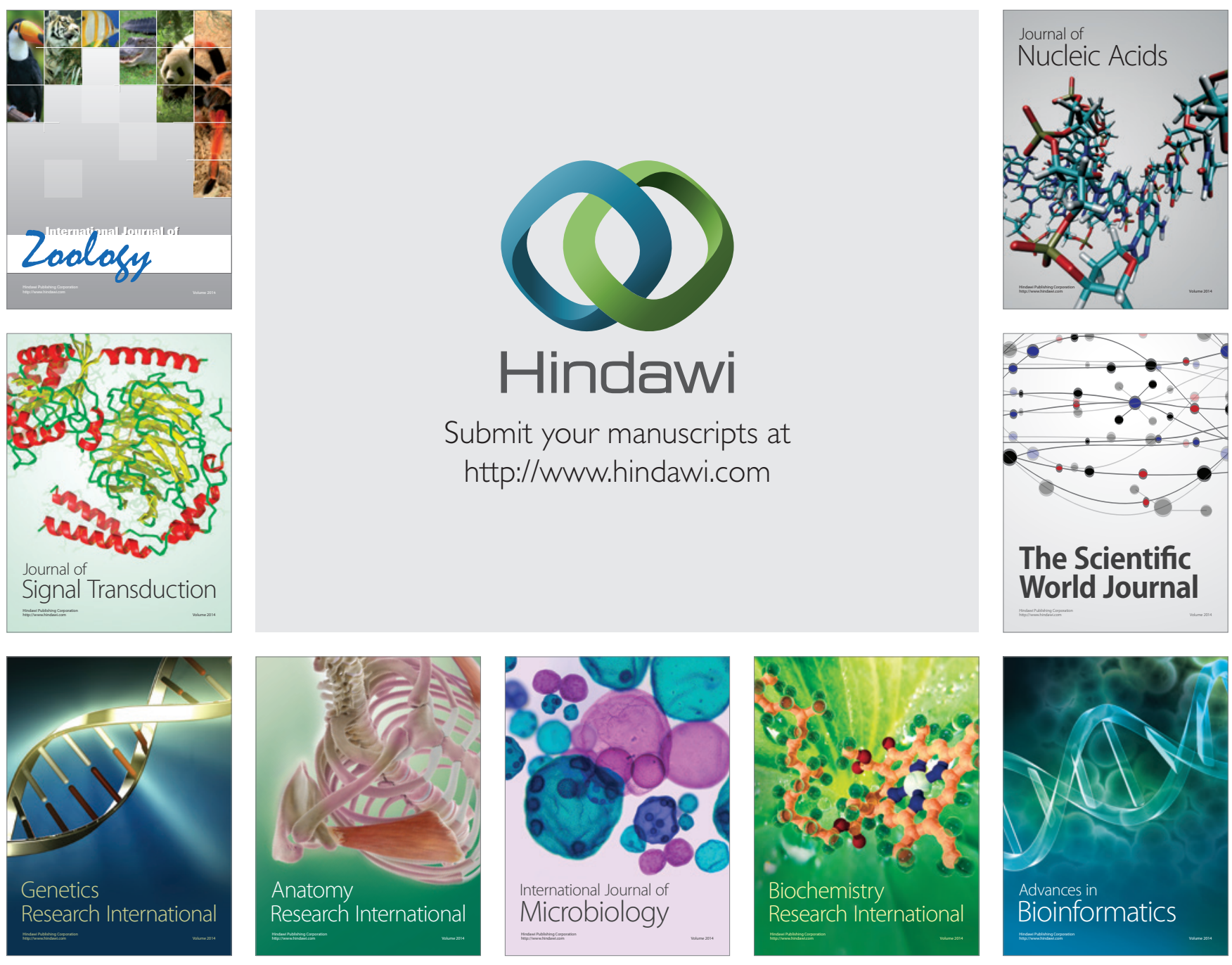

The Scientific World Journal
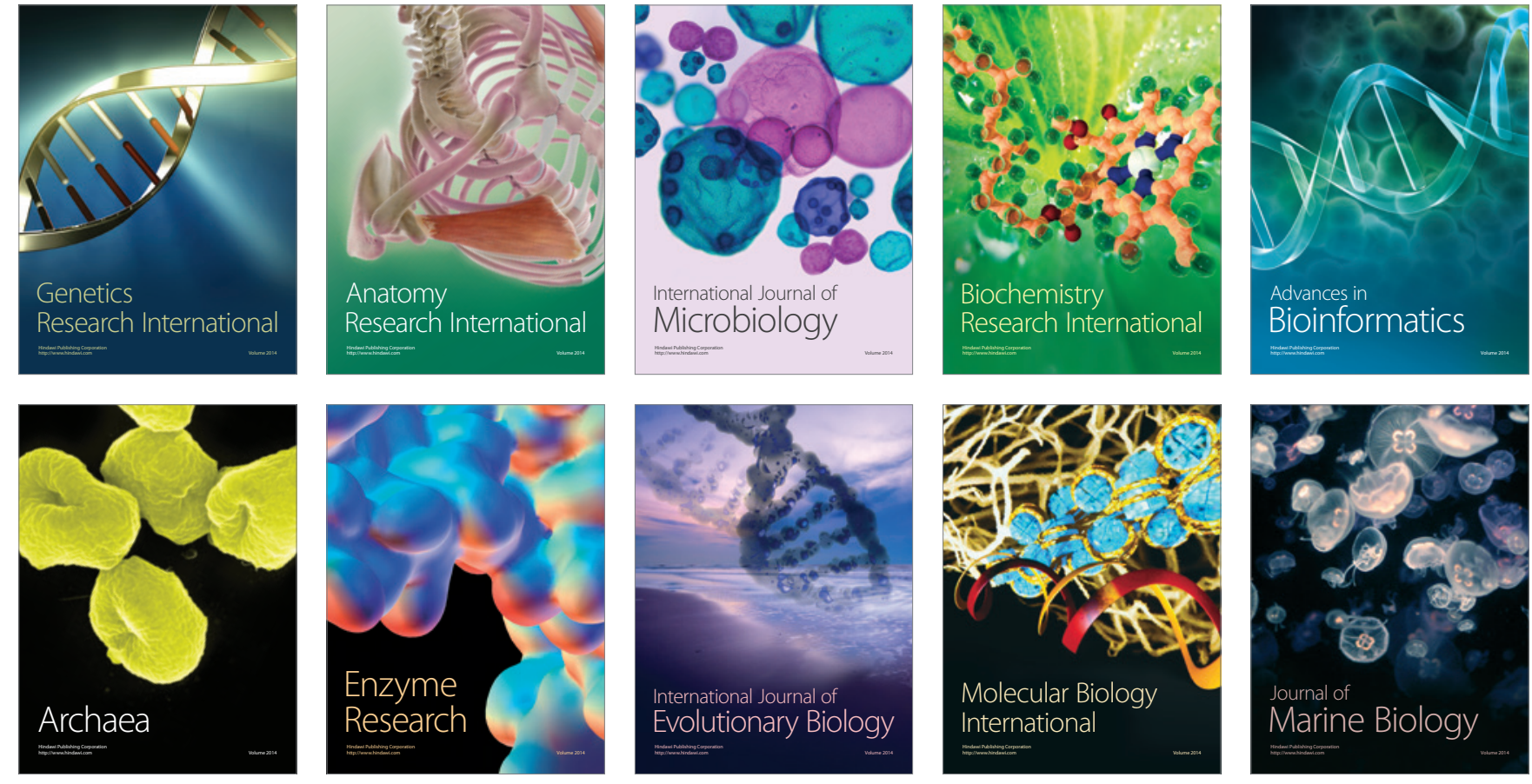